FERMILAB-PUB-96/075-T

BUHEP-96-9

hep-ph/9607213

\title{
Low-Scale Technicolor at the Tevatron
}

\author{
Estia Eichten ${ }^{\dagger}$ \\ Fermi National Accelerator Laboratory \\ P.O. Box 500 Batavia, IL 60510 \\ and \\ Kenneth Lane ${ }^{\ddagger}$ \\ Department of Physics, Boston University \\ 590 Commonwealth Avenue, Boston, MA 02215
}

\begin{abstract}
In multiscale models of walking technicolor, relatively light color-singlet technipions are produced in $q \bar{q}$ annihilation in association with longitudinal $W$ and $Z$ bosons and with each other. The technipions decay as $\pi_{T}^{0} \rightarrow b \bar{b}$ and $\pi_{T}^{+} \rightarrow c \bar{b}$. Their production rates are resonantly enhanced by isovector technirho vector mesons with mass $M_{W}+M_{\pi_{T}} \lesssim$ $M_{\rho_{T}} \lesssim 2 M_{\pi_{T}}$. At the Tevatron, these associated production rates are 1-10 picobarns for $M_{\pi_{T}} \simeq 100 \mathrm{GeV}$. Such a low mass technipion requires topcolor-assisted technicolor to suppress the decay $t \rightarrow \pi_{T}^{+} b$. Searches for $\pi_{T} \pi_{T}$ production will also be rewarding. Sizable rates are expected if $M_{\rho_{T}} \gtrsim 2 M_{\pi_{T}}+10 \mathrm{GeV}$. The isoscalar $\omega_{T}$ is nearly degenerate with $\rho_{T}$ and is expected to be produced at roughly the same rate. The $\omega_{T}$ should have the distinctive decay modes $\omega_{T} \rightarrow \gamma \pi_{T}^{0}$ and $Z \pi_{T}^{0}$.

$7 / 96$

$\dagger$ eichten@fnal.gov
$\ddagger$ lane@buphyc.bu.edu
\end{abstract}


In the standard one-doublet Higgs model of electroweak symmetry breaking, the cross section for production in $\bar{p} p$ collisions at $1.8 \mathrm{TeV}$ of a $100 \mathrm{GeV}$ Higgs boson $H$ in association with $W^{ \pm}$bosons is about $0.15 \mathrm{pb}$. Even lower rates occur in nonminimal Higgs models, including supersymmetric ones. Such small cross sections require luminosities of $1-10 \mathrm{fb}^{-1}$ to detect the signature $W^{ \pm}+H \rightarrow \ell^{ \pm}+\bar{b} b+\mathbb{E}_{T}$ [1]. In this note, we recall that very similar signatures occur in technicolor models at rates that are 30 or more times greater than the Higgs rate, large enough to be observed at the Tevatron now. In our 1989 paper proposing multiscale technicolor [2], we predicted that resonant production via isovector technirho $\left(\rho_{T}\right)$ vector mesons of $W / Z+\pi_{T}$, with $\pi_{T}$ a technipion of mass of $\sim 100 \mathrm{GeV}$ decaying to heavy quarks, would occur at the Tevatron at the level of a few picobarns. Here, we review our proposal and point out that such a low mass for the technipion requires a new scenario such as topcolor-assisted technicolor [3], [4], [5], [6] to accommodate the top quark's large mass and prevent its decay to $\pi_{T}^{+} b$. If the custodial techni-isospin is approximately conserved, as we expect, the isoscalar partner $\omega_{T}$ of the $\rho_{T}$ is nearly degenerate with it and may be produced at a comparable rate. In addition, there may be an isoscalar technipion, $\pi_{T}^{0 \prime}$, close in mass to to $\pi_{T}^{0}$. The main decay modes of $\omega_{T}$ are expected to be $\gamma / Z+\pi_{T}^{0}$ and $\gamma / Z+\pi_{T}^{0 \prime}$, with $\pi_{T}^{0}$ and $\pi_{T}^{0 \prime} \rightarrow \bar{b} b$, providing spectactular signatures at the Tevatron.

Quark and lepton masses in technicolor are generated by broken extended technicolor (ETC) gauge interactions [7], [8], [9]. Because of the conflict between constraints on flavor-changing neutral currents and the magnitude of ETC-generated masses, this classical version of technicolor failed and was replaced a decade ago by "walking" technicolor [10]. In this kind of gauge theory, the strong technicolor coupling $\alpha_{T C}$ runs very slowly for a large range of momenta, possibly all the way up to the ETC scale, which must be several $100 \mathrm{TeV}$ to suppress flavor-changing effects. This slowly-running coupling permits quark and lepton masses as large as a few $\mathrm{GeV}$ to be generated from ETC interactions at this very high scale.

Walking technicolor models require a large number of technifermions in order that $\alpha_{T C}$ runs slowly. These fermions may belong to many copies of the fundamental representation of the technicolor gauge group (here taken to be $S U\left(N_{T C}\right)$ ), to a few higher dimensional representations, or to both. This led us to argue in Ref. [2] that both large 
and fundamental representations participate in electroweak symmetry breaking. 1. The two types of technifermion condense at widely separated scales [13]. The upper scale is set by the weak decay constant $F_{\pi}=246 \mathrm{GeV}$. Technihadrons associated with the lower scale may be so light, we said, that they are within reach of Tevatron collider experiments.

Light-scale technihadrons generally consist of color singlets (discussed in [2]) and nonsinglets (discussed in [14). In this note, we shall be interested in the color singlets. We consider first the lightest isotriplet of technirho vector mesons, $\rho_{T}^{ \pm, 0}$. We will discuss their isoscalar counterpart, $\omega_{T}$, later. The $\rho_{T}$ decay into pairs of isovector technipion states, $\Pi_{T}^{ \pm, 0}$. In general, the latter are mixtures of the longitudinal weak bosons $W_{L}^{ \pm}, Z_{L}^{0}$ and masseigenstate (pseudo-Goldstone) technipions $\pi_{T}^{ \pm}, \pi_{T}^{0}$. In the simplest parameterization, with just one light isotriplet of $\pi_{T},\left|\Pi_{T}\right\rangle=\sin \chi\left|W_{L}\right\rangle+\cos \chi\left|\pi_{T}\right\rangle$, where $\sin \chi=F_{T} / F_{\pi} \ll 1$ and $F_{T}$ is the decay constant of $\Pi_{T}$. The $\rho_{T}$ partial decay rates are given by (assuming no other open decay channels) [15], 16]

$$
\Gamma\left(\rho_{T} \rightarrow \pi_{A} \pi_{B}\right)=\frac{2 \alpha_{\rho_{T}} \mathcal{C}_{A B}^{2}}{3} \frac{p_{A B}^{3}}{M_{\rho_{T}}^{2}},
$$

where $p_{A B}$ is the technipion momentum and $\mathcal{C}_{A B}^{2}=\sin ^{4} \chi, 2 \sin ^{2} \chi \cos ^{2} \chi, \cos ^{4} \chi$ for $\pi_{A} \pi_{B}=W_{L} W_{L}, W_{L} \pi_{T}+\pi_{T} W_{L}, \pi_{T} \pi_{T}$, respectively. For technifermions in the fundamental representation of $S U\left(N_{T C}\right)$, the $\rho_{T} \rightarrow \pi_{T} \pi_{T}$ coupling $\alpha_{\rho_{T}}$ obtained by naive scaling from QCD is given by

$$
\alpha_{\rho_{T}}=2.91\left(\frac{3}{N_{T C}}\right)
$$

In calculations, we take $N_{T C}=4$.

Extended technicolor interactions couple technipions to quarks and leptons with Higgslike couplings. Technipions are then expected to decay into heavy fermions:

$$
\begin{aligned}
& \pi_{T}^{0} \rightarrow \begin{cases}b \bar{b} & \text { if } M_{\pi_{T}}<2 m_{t}, \\
t \bar{t} & \text { if } M_{\pi_{T}}>2 m_{t}\end{cases} \\
& \pi_{T}^{+} \rightarrow \begin{cases}c \bar{b} \text { or } c \bar{s}, \tau^{+} \nu_{\tau} & \text { if } M_{\pi_{T}}<m_{t}+m_{b}, \\
t \bar{b} & \text { if } M_{\pi_{T}}>m_{t}+m_{b} .\end{cases}
\end{aligned}
$$

1 Technicolor models with QCD-like dynamics cannot have a large number of representations because they produce an $S$-parameter that is too large and positive [11]. Of course, such models are already ruled out because they have flavor-changing neutral currents that are too large [9], the problem that motivated walking technicolor. The arguments in [11] are based on scaling from QCD and on chiral perturbation theory. They fail or are questionable in walking technicolor models; see Ref. 12]. 
Now - and this is the important feature of walking technicolor - technipion masses are enhanced by renormalizations that are so large that the $\rho_{T} \rightarrow \pi_{T} \pi_{T}$ channels may be closed or strongly suppressed. Thus, technirho production at the Tevatron can lead to all of the processes

$$
\begin{aligned}
& q \bar{q}^{\prime} \rightarrow W^{ \pm} \rightarrow \rho_{T}^{ \pm} \rightarrow W_{L}^{ \pm} Z_{L}^{0} ; \quad W_{L}^{ \pm} \pi_{T}^{0}, \pi_{T}^{ \pm} Z_{L}^{0} ; \quad \pi_{T}^{ \pm} \pi_{T}^{0} \\
& q \bar{q} \rightarrow \gamma, Z^{0} \rightarrow \rho_{T}^{0} \rightarrow W_{L}^{+} W_{L}^{-} ; \quad W_{L}^{ \pm} \pi_{T}^{\mp} ; \quad \pi_{T}^{+} \pi_{T}^{-}
\end{aligned}
$$

occurring at comparable rates despite the small angle $\chi$.

To illustrate our points, we shall take $M_{\pi_{T}^{ \pm}} \simeq M_{\pi_{T}^{0}} \simeq 110 \mathrm{GeV}$ and vary $M_{\rho_{T}^{ \pm}} \simeq M_{\rho_{T}^{0}}$ in our calculations. Note that, since $\rho_{T}$ has $I=1$, these resonant processes do not lead to $Z^{0} \pi_{T}^{0} \rightarrow \ell^{+} \ell^{-} / \nu \bar{\nu}+b \bar{b}$ final states. Such events must originate from $\omega_{T}$, as we discuss later. Technirho events with a $Z^{0}$ and two heavy quark jets have one $b$-jet and one $c$-jet.

The Drell-Yan processes in Eq. (四) have $\mathcal{O}\left(\alpha^{2}\right)$ cross sections and are unobservably small compared to backgrounds unless the technirho resonances are not far above threshold, roughly $M_{W}+M_{\pi_{T}} \lesssim M_{\rho_{T}} \lesssim 2 M_{\pi_{T}}$. This condition is favored by multiscale technicolor. We estimate the $\rho_{T} \rightarrow \pi_{A} \pi_{B}$ subprocess cross sections by vector meson dominance, taking the $\gamma, Z, W \rightarrow \rho_{T}$ couplings from the condition that they reproduce $\gamma, Z, W \rightarrow \pi_{A} \pi_{B}$ at zero energy. We obtain [2]

$$
\frac{d \hat{\sigma}\left(q_{i} \bar{q}_{j} \rightarrow \rho_{T}^{ \pm, 0} \rightarrow \pi_{A} \pi_{B}\right)}{d z}=\frac{\pi \alpha^{2} p_{A B}^{3}}{3 \hat{s}^{5 / 2}} \frac{M_{\rho_{T}}^{4}\left(1-z^{2}\right)}{\left(\hat{s}-M_{\rho_{T}}^{2}\right)^{2}+\hat{s} \Gamma_{\rho_{T}}^{2}} A_{i j}^{ \pm, 0}(\hat{s}) \mathcal{C}_{A B}^{2}
$$

where $\hat{s}$ is the subprocess energy, $z=\cos \theta$ is the $\pi_{A}$ production angle, and $\Gamma_{\rho_{T}}$ is the energy-dependent total width. Ignoring Kobayashi-Maskawa mixing angles, the factors $A_{i j}^{ \pm, 0}=\delta_{i j} A^{ \pm, 0}$ are

$$
\begin{aligned}
A^{ \pm} & =\frac{1}{4 \sin ^{4} \theta_{W}}\left(\frac{\hat{s}}{\hat{s}-M_{W}^{2}}\right)^{2}, \\
A^{0} & =\left[Q_{i}+\frac{2 \cos 2 \theta_{W}}{\sin ^{2} 2 \theta_{W}}\left(T_{3 i}-Q_{i} \sin ^{2} \theta_{W}\right)\left(\frac{\hat{s}}{\hat{s}-M_{Z}^{2}}\right)\right]^{2} \\
& +\left[Q_{i}-\frac{2 Q_{i} \cos 2 \theta_{W} \sin ^{2} \theta_{W}}{\sin ^{2} 2 \theta_{W}}\left(\frac{\hat{s}}{\hat{s}-M_{Z}^{2}}\right)\right]^{2} .
\end{aligned}
$$

2 Note that $Z+\pi_{T}$ events with a lost lepton can end up in the $W+\pi_{T}$ sample, while $W+\pi_{T}$ with a lost lepton may be counted as $Z(\rightarrow \nu \bar{\nu})+\pi_{T}$. 
Here, $Q_{i}$ and $T_{3 i}$ are the electric charge and third component of weak isospin for quark $q_{i L, R}$. In these processes, the $W+$ dijet and $Z+$ dijet invariant masses exhibit a narrow peak not far above $200 \mathrm{GeV}$. This peak will be smeared by energy resolutions, but it should be narrower than expected from continuum production of $W / Z+H$.

Our final observation is that, after all this, ordinary multiscale technicolor cannot accommodate the top quark. Its mass of $m_{t} \simeq 175 \mathrm{GeV}$ [17] is much too large to be generated by ETC coupling of the top quark to the low-scale technifermions [14]. Furthermore, there cannot be a charged technipion as light as $110 \mathrm{GeV}$ for, then, the top quark would tend to decay into it. Taking the coupling of $\pi_{T}^{+}$to $\bar{t}_{L} b_{R}$ to be $\sqrt{2} m_{t}^{E T C} / F_{T}$, where $m_{t}^{E T C}$ is the ETC contribution to the top-quark mass, the top decay rate to $\pi_{T}^{+} b$ is

$$
\Gamma\left(t \rightarrow \pi_{T}^{+} b\right)=\frac{\left(m_{t}^{2}-M_{\pi_{T}}^{2}\right)^{2}}{16 \pi m_{t} F_{T}^{2}}\left(\frac{m_{t}^{E T C}}{m_{t}}\right)^{2} .
$$

For $m_{t}^{E T C} \simeq m_{t}$ and $F_{T}=40 \mathrm{GeV}$, a typical value in multiscale models, $\Gamma\left(t \rightarrow \pi_{T}^{+} b\right) \simeq$ $25 \mathrm{GeV} \simeq 15 \Gamma\left(t \rightarrow W^{+} b\right)$; this is ruled out [18]. Topcolor-assisted technicolor (TC2) resolves these problems.

In TC2 [5], as in top-condensate models of electroweak symmetry breaking [3], [4], the large top quark mass is generated by strong "topcolor" gauge interactions. Thus, there can be low-scale technifermions and the ETC scale can be $\mathcal{O}(100 \mathrm{TeV})$ for all fermions. Then, $m_{t}^{E T C}$ is only a few $\mathrm{GeV}$, so that the branching fraction $B\left(t \rightarrow \pi_{T}^{+} b\right)$ is small.

Preliminary models of topcolor-assisted technicolor were developed in Ref. [6]. They differ from multiscale technicolor models in that they do not contain technifermions in higher representations and the associated widely separated scales. However, there are many copies of the fundamental representation (some of which also transform under color or topcolor $S U(3))$. Thus, the net effect is the same; ignoring $S U(3)$ effects, $F_{T} \simeq F_{\pi} / \sqrt{N_{D}}$, where $N_{D}$ is the number of technifermion weak isodoublets. In the model of the second paper in [6], $N_{D}=9$, so that $F_{T}=82 \mathrm{GeV}$ (or somewhat less because of color effects) for the lightest color-singlet technipions. ${ }^{1}$

We have used Eq. (5) with $\sin \chi=\frac{1}{3}$ to compute the $\rho_{T}$ production cross sections for $M_{\pi_{T}}=110 \mathrm{GeV}$ and $M_{\rho_{T}}=195-250 \mathrm{GeV}$. The individual decay channel cross sections

3 Top-pions $\pi_{t}$ arising from top-quark condensation do couple to $m_{t}$. Their mass arises from the ETC contribution: $M_{\pi_{t}}^{2} \simeq m_{t}^{E T C}\langle\bar{t} t\rangle / F_{t}^{2}$, where $F_{t} \simeq 70 \mathrm{GeV}$ [5]. This is sufficient to make $M_{\pi_{t}} \gtrsim m_{t}$. Mixing of top-pions with technipions is expected to be small, of order $\langle\bar{t} t\rangle /\langle\bar{T} T\rangle_{E T C} \simeq$ $\Lambda_{T C} / \Lambda_{E T C} \lesssim 10^{-2}$.19. 
are shown for $p \bar{p}$ collisions at $\sqrt{s}=1.8 \mathrm{TeV}$ in Fig. 1 (multiplied by a $K$-factor of 1.5 , appropriate for Drell-Yan processes at the Tevatron). No cuts were put on the technipion directions. These plots illustrate several features we expect to be general:

- Except near $W \pi_{T}$ threshold, the increase in $W Z$ and $W W$ production is small.

○ The inclusive $W \pi_{T}$ rate is $5-10 \mathrm{pb}$ and the $Z \pi_{T}$ rate is $1-3 \mathrm{pb}$ for $M_{\pi_{T}}+M_{W} \lesssim$ $M_{\rho_{T}} \lesssim 2 M_{\pi_{T}}$. The ratio $\sigma\left(W_{L} \pi_{T}\right) / \sigma\left(Z_{L} \pi_{T}\right)=2-3$ is about the same as expected for $\sigma(W H) / \sigma(Z H)$. Because of threshold singularities, these rates can be changed significantly by modest isospin splittings in the technihadron masses. The cross sections increase by $15-20 \%$ when the Tevatron energy is increased to $2 \mathrm{TeV}$.

- Because the technirho is narrow, the total $p p^{ \pm}$cross section at $\sqrt{s}$ into the channel $\pi_{A} \pi_{B}$ is

$$
\begin{aligned}
\sigma\left(p p^{ \pm}\right. & \left.\rightarrow \rho_{T} \rightarrow \pi_{A} \pi_{B}\right) \simeq \frac{2 \pi^{2}}{3 s} \sum_{i, j} \frac{\Gamma\left(\rho_{T} \rightarrow q_{i} \bar{q}_{j}\right) \Gamma\left(\rho_{T} \rightarrow \pi_{A} \pi_{B}\right)}{M_{\rho_{T}} \Gamma_{\rho_{T}}} \\
& \times \int d \eta_{B}\left\{f_{q_{i}}^{p}\left(\frac{M_{\rho_{T}}}{\sqrt{s}} e^{\eta_{B}}\right) f_{\bar{q}_{j}}^{p^{ \pm}}\left(\frac{M_{\rho_{T}}}{\sqrt{s}} e^{-\eta_{B}}\right)+\left(q_{i} \leftrightarrow \bar{q}_{j}\right)\right\},
\end{aligned}
$$

where $f_{q_{i}}^{p^{ \pm}}$is the $q_{i}$ distribution function in $p^{ \pm}$at $Q^{2}=M_{\rho_{T}}^{2}$. At the Tevatron, these distributions favor $\rho_{T}^{ \pm}$production over $\rho_{T}^{0}$ by a factor of $2-3$ over the range of $M_{\rho_{T}}$ considered.

○ For $M_{\rho_{T}} \geq 2 M_{\pi_{T}}+10 \mathrm{GeV}$, the dominant process is $\pi_{T}^{ \pm} \pi_{T}^{0}$ production. The crossover point depends to some extent on the suppression factor tan $\chi$, but we don't expect it to be much different from this. A search for the $\pi_{T}^{ \pm} \pi_{T}^{0}$ channel will be rewarding.

Finally, we turn to the $\omega_{T}$. The walking technicolor enhancement of technipion masses almost certainly closes off the isospin-conserving decay $\omega_{T} \rightarrow \Pi_{T}^{+} \Pi_{T}^{-} \Pi_{T}^{0}$. Even the triplysuppressed mode $W_{L}^{+} W_{L}^{-} Z_{L}$ has little or no phase space for the $M_{\omega_{T}}$-range we are considering. Thus, we expect the decays $\omega_{T} \rightarrow \gamma \Pi_{T}^{0}, Z \Pi_{T}^{0}$, and $\Pi_{T}^{+} \Pi_{T}^{-}$. When written in terms of mass eigenstates, these modes are $\omega_{T} \rightarrow \gamma \pi_{T}^{0}, \gamma Z_{L}, Z \pi_{T}^{0}, Z Z_{L} ; \gamma \pi_{T}^{0 \prime}, Z \pi_{T}^{0 \prime}$; and $W_{L}^{+} W_{L}^{-}, \pi_{T}^{ \pm} W_{L}^{\mp}, \pi_{T}^{+} \pi_{T}^{-}$. . It is not possible to estimate the relative magnitudes of the decay amplitudes without an explicit model of the $\omega_{T}$ 's constituent technifermions. Judging from the decays of the ordinary $\omega$, we expect $\omega_{T} \rightarrow \gamma \pi_{T}^{0}\left(\pi_{T}^{0 \prime}\right), Z \pi_{T}^{0}\left(\pi_{T}^{0 \prime}\right)$ to be dominant, with the former mode favored by phase space.

4 The modes $\omega_{T} \rightarrow \gamma Z_{L}, Z Z_{L}$ were considered for a one-doublet technicolor model in Ref. [20]. We have estimated the rates for the isospin-violating decays $\rho_{T} \rightarrow \gamma \pi_{T}^{0}, Z \pi_{T}^{0}$ and find them to be negligible unless the mixing angle $\chi$ is very small. 
The $\omega_{T}$ is produced in hadron collisions just as the $\rho_{T}^{0}$, via its vector-meson-dominance coupling to $\gamma$ and $Z^{0}$. For $M_{\omega_{T}} \simeq M_{\rho_{T}}$, the $\omega_{T}$ production cross section should be approximately $\left|Q_{U}+Q_{D}\right|^{2}$ times the $\rho_{T}^{0}$ rate, where $Q_{U, D}$ are the electric charges of the $\omega_{T}$ 's constituent technifermions. The principal signatures for $\omega_{T}$ production, then, are $\gamma+\bar{b} b$ and $\ell^{+} \ell^{-}($or $\nu \bar{\nu})+b \bar{b}$, with $M_{\bar{b} b}=M_{\pi_{T}}$.

The model of color-singlet technihadron production we have discussed here is an oversimplification, but one that captures some of the essence of modern models of technicolor. As we did in Ref. [2], we urge a search for $W / Z+\pi_{T} \rightarrow$ isolated high- $p_{T}$ leptons + heavy quark jets. If such events are found, the $W / Z+j j$ mass spectrum should exhibit a narrow $\rho_{T}$ peak consistent with resolution. Sidebands $-M_{W j j}$ with $M_{j j}$ outside the " $M_{\pi_{T}}$ " bins - should not exhibit the peak unless it turns out to be kinematic in nature. Because of the quadratic ambiguity in reconstructing the $W$ in its $\ell \nu$ decay, it seems best to plot the low-mass solution versus the high-mass one. We also urge a search for $\pi_{T} \pi_{T}$ production. This may not be possible now, but a search with the high-luminosity data of Tevatron Run II should be conclusive. If a $\pi_{T}$ candidate is found, it will be important to determine whether $c$-quarks as well as $b$-quarks occur in its decay. Higher luminosity and more robust heavy-flavor tagging can make this possible. Finally, there should be an isoscalar $\omega_{T}$ nearly degenerate with $\rho_{T}^{0}$, generically produced with a comparable cross section, and with spectacular $\gamma \bar{b} b$ and $Z \bar{b} b$ decay signatures. If these technihadrons are found, they will be just the first of a very large family.

EE's research is supported by the Fermi National Accelerator Laboratory, which is operated by Universities Research Association, Inc., under Contract No. DE-AC0276CHO3000. KL's research is supported in part by the Department of Energy under Grant No. DE-FG02-91ER40676. We gratefully acknowledge the hospitality of the Aspen Center for Physics where this work was completed. 


\section{References}

[1] "Report of the tev_2000 Study Group on Future Electroweak Physics at the Tevatron", edited by D. Amidei and R. Brock, DØ Note 2589 and CDF Note 3177, (1995).

[2] K. Lane and E. Eichten, Phys. Lett. B222, 274 (1989)

[3] Y. Nambu, in New Theories in Physics, Proceedings of the XI International Symposium on Elementary Particle Physics, Kazimierz, Poland, 1988, edited by Z. Adjuk, S. Pokorski and A. Trautmann (World Scientific, Singapore, 1989); Enrico Fermi Institute Report EFI 89-08 (unpublished);

V. A. Miransky, M. Tanabashi and K. Yamawaki, Phys. Lett. 221B, 177 (1989); Mod. Phys. Lett. A4, 1043 (1989);

W. A. Bardeen, C. T. Hill and M. Lindner, Phys. Rev. D41, 1647 (1990).

[4] C. T. Hill, Phys. Lett. 266B, 419 (1991) ;

S. P. Martin, Phys. Rev. D45, 4283 (1992); ibid D46, 2197 (1992); Nucl. Phys. B398, 359 (1993); M. Lindner and D. Ross, Nucl. Phys. B370, 30 (1992);

R. Bönisch, Phys. Lett. 268B, 394 (1991);

C. T. Hill, D. Kennedy, T. Onogi, H. L. Yu, Phys. Rev. D47, 2940 (1993).

[5] C. T. Hill, Phys. Lett. 345B, 483 (1995).

[6] K. Lane and E. Eichten, Phys. Lett. B352, 382 (1995) ;

K. Lane, Boston University Preprint BUHEP-96-2, hep-ph/9602221, submitted to Physical Review D.

[7] S. Weinberg, Phys. Rev. D19, 1277 (1979);

L. Susskind, Phys. Rev. D20, 2619 (1979).

[8] S. Dimopoulos and L. Susskind, Nucl. Phys. B155, 237 (1979).

[9] E. Eichten and K. Lane, Phys. Lett. 90B, 125 (1980).

[10] B. Holdom, Phys. Rev. D24, 1441 (1981); Phys. Lett. 150B, 301 (1985);

T. Appelquist, D. Karabali and L. C. R. Wijewardhana, Phys. Rev. Lett. 57, 957 (1986); T. Appelquist and L. C. R. Wijewardhana, Phys. Rev. D36, 568 (1987);

K. Yamawaki, M. Bando and K. Matumoto, Phys. Rev. Lett. 56, 1335 (1986) ;

T. Akiba and T. Yanagida, Phys. Lett. 169B, 432 (1986).

[11] A. Longhitano, Phys. Rev. D22 (1980) 1166; Nucl. Phys. B188, (1981) 118;

R. Renken and M. Peskin, Nucl. Phys. B211 (1983) 93;

B. W. Lynn, M. E. Peskin and R .G. Stuart, in Trieste Electroweak 1985, 213;

M. Golden and L.Randall, Nucl. Phys. B361 (1990) 3;

B. Holdom and J. Terning, Phys. Lett. B247 (1990) 88;

M. E. Peskin and T. Takeuchi, Phys. Rev. Lett. 65 (1990) 964;

A. Dobado, D. Espriu and M. J. Herrero, Phys. Lett. B255 (1990) 405;

H. Georgi, Nucl. Phys. B363 (1991) 301. 
[12] K. Lane, Proceedings of the 27th International Conference on High Energy Physics, edited by P. J. Bussey and I. G. Knowles, Vol. II, p. 543, Glasgow, June 20-27, 1994.

[13] S. Raby, S. Dimopoulos and L. Susskind, Nucl. Phys. B169, 373 (1980).

[14] K. Lane and M. V. Ramana, Phys. Rev. D44, 2678 (1991).

[15] S. Dimopoulos, S. Raby and G. Kane, Nucl. Phys. B182, 77 (1981).

[16] E. Eichten, I. Hinchliffe, K. Lane and C. Quigg, Rev. Mod. Phys. 56, 579 (1984); Phys. Rev. D34, 1547 (1986).

[17] F. Abe, et al., The CDF Collaboration, Phys. Rev. Lett. 73, 225 (1994); Phys. Rev. D50, 2966 (1994); Phys. Rev. Lett. 74, 2626 (1995) ;

S. Abachi, et al., The DØ Collaboration, Phys. Rev. Lett. 74, 2632 (1995).

[18] J. Incandela, Proceedings of the 10th Topical Workshop on Proton-Antiproton Collider Physics, Fermilab, edited R. Raja and J. Yoh, p. 256 (1995).

[19] B. Balaji, "Top-Pion-Technipion Mixing in Natural Topcolor-Assisted Technicolor", Boston University preprint in preparation.

[20] R. S. Chivukula and M. Golden, Phys. Rev. D41, 2795 (1990). 


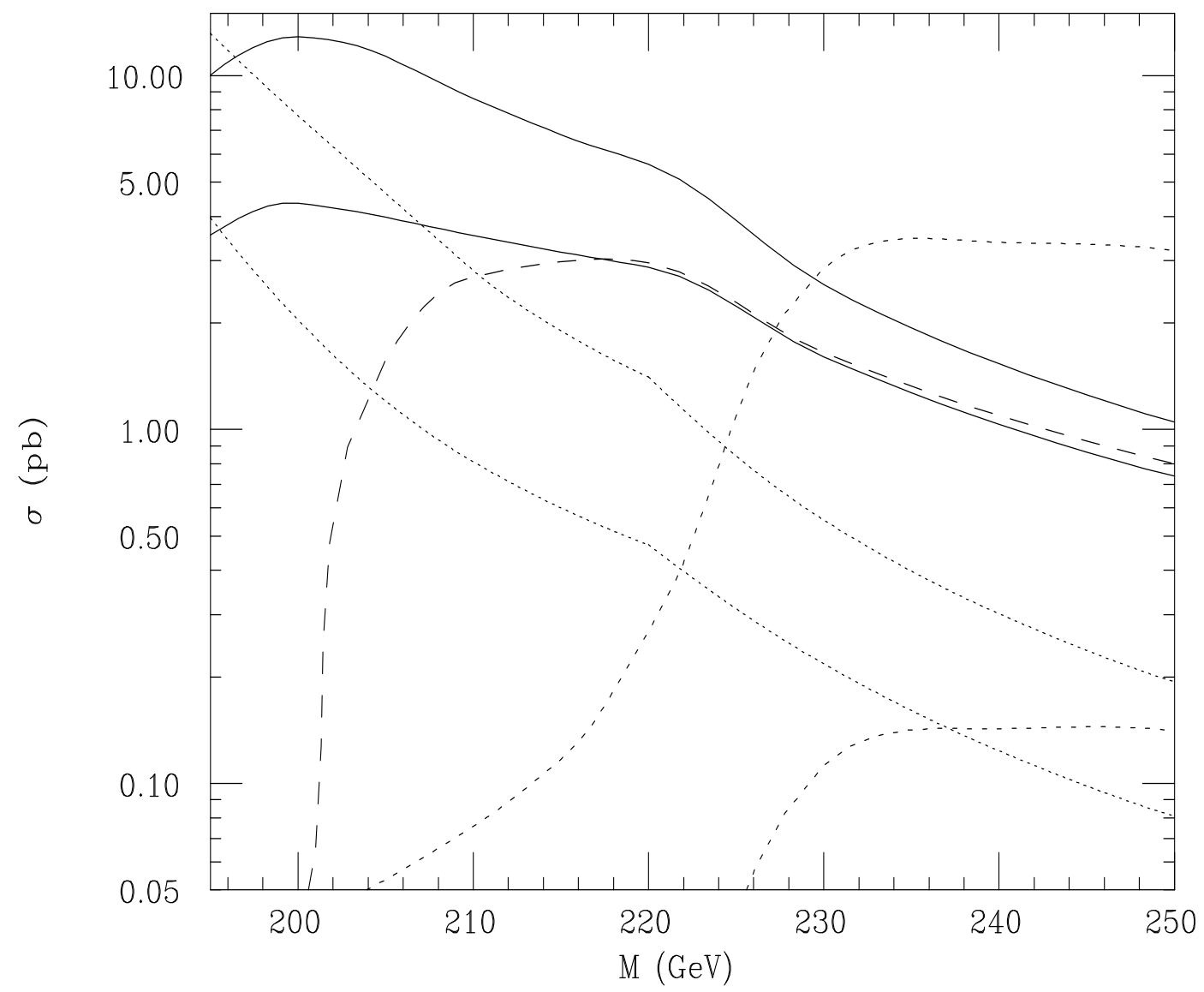

Figure 1. Total $W W, W \pi_{T}$ and $\pi_{T} \pi_{T}$ cross sections in $\bar{p} p$ collisions at $1.8 \mathrm{TeV}$, as a function of $M_{\rho_{T}}$ for $M \pi_{T}=110 \mathrm{GeV}$. The model described above Eq. (1) is used with $\sin \chi=\frac{1}{3}$. The curves are $W^{ \pm} Z^{0}$ (upper dotted) and $W^{+} W^{-}$(lower dotted); $W^{ \pm} \pi_{T}^{0}$ (upper solid), $W^{ \pm} \pi_{T}^{\mp}$ (lower solid), and $Z^{0} \pi_{T}^{ \pm}$(long dashed); $\pi_{T}^{ \pm} \pi_{T}^{0}$ (upper short dashed) and $\pi_{T}^{+} \pi_{T}^{-}$(lower short dashed). EHLQ set 1 distribution functions [16] were used and cross sections were multiplied by a $K$-factor of 1.5 , as appropriate for Drell-Yan processes. 